\title{
Isotonic mechanics of a pharyngeal dilator muscle and diaphragm in the rat before and after fatigue
}

\author{
P. Attal*, C. Coirault**, D. Chemla ${ }^{+}$, F-X. Blanc**, P. Rocher*, J-C. Pourny**, \\ S. Bobin*, Y. Lecarpentier ${ }^{+}$
}

Isotonic mechanics of a pharyngeal dilator muscle and diaphragm in the rat before and after fatigue. P. Attal, C. Coirault, D. Chemla, F-X. Blanc, P. Rocher, J-C. Pourny, S. Bobin, Y. Lecarpentier. C) ERS Journals Ltd 2000.

ABSTRACT: Pharyngeal and diaphragm muscles contract and relax in synergy, which is why it was decided to compare their mechanical performance throughout the overall load continuum. The effects of fatigue were also studied.

The isotonic mechanics of rat sternohyoid $(\mathrm{SH} ; \mathrm{n}=10)$ and diaphragm $(\mathrm{D} ; \mathrm{n}=10)$ were investigated in vitro. Force and length were measured in muscles contracting from zero load up to isometry. Maximum isometric tension $(P \max )$, peak mechanical work ( $W$ max), maximum unloaded shortening velocity (vZL) and mechanical efficiency (effmax) were recorded. Data were obtained both at baseline and after fatigue.

SH muscles had a lower $P \max \left(96.0 \pm 13.7\right.$ versus $\left.119.5 \pm 22.7 \mathrm{mN} . \mathrm{mm}^{-2} ; \mathbf{p}<0.05\right)$, a lower $W_{\max }\left(5.5 \pm 1.2\right.$ versus $\left.8.0 \pm 2.1 \mathrm{~mJ}^{-1} ; \mathrm{p}<0.01\right)$, a lower effmax $(56.0 \pm 6.9$ versus $62.6 \pm 5.8 \% ; \mathrm{p}<0.05)$ and a higher vzL $\left(4.8 \pm 0.4\right.$ versus $3.4 \pm 0.4$ initial length $(\mathrm{Lo}) . \mathrm{s}^{-1}$; $\mathrm{p}<0.001)$ than $\mathrm{D}$ muscles. $W_{\max }$ occurred at a higher relative load in $\mathrm{SH}\left(40 \% P_{\max }\right)$ than in D (30\% P $\max )$. Fatigue did not modify effmax in SH muscles, whereas it significantly improved effmax in D muscles.

These findings suggest that under control conditions, economy of force generation was less efficient in sternohyoid than in diaphragm muscles. Fatigue in sternohyoid muscles induced unfavourable mechanical behaviour. This may partly explain pharyngeal dilator muscle failure in the presence of increased loads. Whether these findings are relevant to human sleep apnoea syndrome has yet to be determined.

Eur Respir J 2000; 15: 308-313.
*Service d'ORL et de Chirurgie CervicoFaciale and ${ }^{+}$Service de Physiologie Cardiovasculaire et Respiratoire, UER Paris XI, Hôpital de Bicêtre, Assistance Publique - Hôpitaux de Paris. 94275 Le Kremlin-Bicêtre and **INSERM 451Loa-Ensta-Ecole Polytechnique, 91125 Palaiseau, France.

Correspondence: Y. Lecarpentier

Service de Physiologie Cardiovasculaire et Respiratoire

Centre Hospitalier et Universitaire de Bicêtre

78 rue du Général Leclerc

F 94275 Le Kremlin Bicêtre cedex

France

Fax: 33169319996

Keywords: Fatigue mechanical efficiency mechanical work skeletal muscle upper airways

Received: March 311999

Accepted after revision August 251999
Pharyngeal muscles can be considered as belonging to the respiratory muscles [1]. Synchronized activity of the pharyngeal muscles and diaphragm (D) is observed in humans and animals [2]. Pharyngeal dilator muscles exhibit phasic activity during inspiration [3-5], whereas pharyngeal constrictor muscles exhibit phasic activity during expiration [6]. In synergy with the D, the pharyngeal muscles contribute to upper airway patency during breathing in awake and sleeping subjects [7]. The negative pressure generated in the upper airways by D contraction is one of the most important factors involved in the stimulation of pharyngeal dilator muscles [8], which promotes opening of the upper airways during inspiration [7]. The activity of pharyngeal muscles has also been studied in sleep apnoea syndrome, in which repeated occlusions of the upper airways occur during sleep. Pharyngeal occlusions seem to be associated with a relative decline in the activity of pharyngeal dilator muscles [7]. The end of these occlusions is associated with large bursts of dilator muscle activity. The acute effects of these repetitive episodes of pharyngeal dilator muscle recruitment on the mechanical properties of these muscles remain unknown.

The relative mechanical properties of pharyngeal and D muscles under control conditions and during fatigue may thus have important pathophysiological implications. The mechanical properties of pharyngeal dilator muscles have been documented both in situ and in vitro [9-11]. Pharyngeal muscles generally have shorter contraction times and lower twitch to tetanic tension ratios than the D. These studies were performed under isometric conditions, in which no shortening of the pharyngeal muscles occurs. As pharyngeal muscles contract against various levels of load, their ability to shorten and to produce mechanical work $(W)$ needs to be documented.

The aim of the present study was three-fold: 1) to document for the first time the isotonic mechanical properties of sternohyoid ( $\mathrm{SH}$ ) muscle, a pharyngeal dilator muscle; 2) to compare the effects of increasing load on working $\mathrm{SH}$ and D muscles; and 3) to study the mechanical consequences of fatigue on $\mathrm{SH}$ and $\mathrm{D}$ muscles. The hypothesis that $\mathrm{SH}$ and $\mathrm{D}$ muscles have different isotonic mechanical properties at baseline and during fatigue was tested.

\section{Materials and methods}

In 20 male adult Wistar rats, experiments were conducted on strips of SH $(n=10)$ and $\mathrm{D}(\mathrm{n}=10)$ muscles. Animal care was in keeping with the recommendations of the Helsinki Declaration. Animals were anaesthetized with 
pentobarbital sodium (60 mg. $\mathrm{kg}^{-1}$ intraperitoneally). The rationale for studying SH muscles was as follows. Firstly, the contribution of SH muscles to safeguarding upper airway patency is well documented [5]. Secondly, it is possible to keep bony origin and tendon intact, thus preventing fibre damage, which is not the case for other pharyngeal dilator muscles (personal unpublished observation) [11].

SH strips were removed via a median cervicotomy from the mandible to the manubrium and after separation of the submaxillary glands. First, the suprahyoidian muscular attachments were cut, leaving the hyoid bone attached to the infrahyoid muscles. The hyoid bone was cut medially and then the two SH muscles separated along the middle line as far as the inferior end of the muscles, requiring a sternotomy. A strip of SH muscle (1-2 mm width) was obtained by cutting one of the two SH muscles laterally. At the upper end of the strip, a piece of hyoid bone was left attached (hyoid end) and at the lower end a piece of sternum (sternum end). Following a median laparotomy, a strip was cut out from the ventral costal D muscle in situ. One strip (i.e. D or SH muscle strip) was obtained per animal. Each muscle strip was vertically suspended in a bath containing the following Krebs-Henseleit solution (in $\mathrm{mM}): 118 \mathrm{NaCl}, 4.7 \mathrm{KCl}, 1.2 \mathrm{MgSO}_{4} .7 \mathrm{H}_{2} \mathrm{O}, 1.1 \mathrm{KH}_{2} \mathrm{PO}_{4}$, $24 \mathrm{NaHCO}_{3}, 2.5 \mathrm{CaCl}_{2} .6 \mathrm{H}_{2} \mathrm{O}$ and 4.5 glucose. The solution was bubbled with $95 \% \mathrm{O}_{2} / 5 \% \mathrm{CO}_{2}$ and maintained at $22^{\circ} \mathrm{C}(\mathrm{pH}$ 7.4). The costal end of the $\mathrm{D}$ strip and the sternum end of the SH strip were held in a stationary clip at the bottom of the bath, and the upper ends were held in a spring clip attached to an electromagnetic lever system.

Muscles were stimulated by means of two platinum electrodes in twitch mode ( 5 stimulations $\cdot \mathrm{min}^{-1}$ with a rectangular pulse of $1 \mathrm{~ms}$ duration). Muscles were also stimulated supramaximally under tetanic conditions as follows: electrical stimulus $1 \mathrm{~ms}$; stimulation frequency $50 \mathrm{~Hz}$; train duration $200 \mathrm{~ms}$; train frequency 5 trains. $\mathrm{min}^{-1}$. At $22^{\circ} \mathrm{C}$, the $50-\mathrm{Hz}$ stimulation frequency yielded the tetanic maximum isometric tension $(P \max )$ for both the SH pharyngeal muscle and the D [12]. Experiments were carried out at the initial resting length corresponding to the apex of the initial length/active tension curve (L0). At the end of the study, the cross-sectional area (in $\mathrm{mm}^{2}$ ) was calculated from the ratio of fresh muscle weight to muscle length at L0, assuming a muscle density of 1 .

\section{The electromagnetic system}

The load applied to the muscle was determined by means of a servocontrolled current through the coil of an electromagnet. The amplitude of force measurement ranged $0-140 \mathrm{mN}$. The error of the measured force was $<0.1 \%$. The equivalent moving mass of the whole system was 155 $\mathrm{mg}$ and its compliance was $0.2 \mu \mathrm{m} \cdot \mathrm{mN}^{-1}$. Muscle shortening displaced the lever, thereby modulating the light intensity received by a photoelectric transducer. The linearity of the system ranged $0-5 \mathrm{~mm}$ muscle shortening, and the error was $<0.5 \%$ of full-scale deflection. All analyses were based on digital recordings obtained using a microcomputer. Two signals, tension (i.e. force $\cdot \mathrm{mm}^{-2}$ ) and length, were recorded. Software for calculating all of the mechanical parameters was developed in the authors' laboratory.

\section{Mechanical indices}

Muscles were studied: 1) under full isometric conditions, in which total isometric muscle tension reflects both the total number and the single force of actomyosin interactions [13-15]; 2) at zero load, at which maximum unloaded shortening velocity (vZL) is related to crossbridge kinetics and particularly to the rate constant for cross-bridge detachment [13]; and 3) from zero load up to isometry, where the characteristics of the tension/velocity relationship (Hill's hyperbola), peak mechanical work $\left(W_{\max }\right)$ and peak mechanical efficiency (effmax) indirectly reflect the energetic processes within the muscle.

Maximum isometric tension and unloaded shortening velocity. Classic mechanical parameters characterizing the contraction phase were measured from two contractions under twitch and tetanic conditions. The first contraction was loaded with preload only and was clamped to zero load immediately after the electrical stimulus, using the zero load clamp technique [16]. The second contraction was fully isometric. The shortening length and tension/time curves were recorded simultaneously. The following indices were used to assess muscle performance during the contraction phase: 1) measured vZL (in L $0 \cdot \mathrm{s}^{-1}$ ) (isotonic mode); and 2) $P \max \left(\right.$ in $\mathrm{mN} \cdot \mathrm{mm}^{-2}$ ) (isometric mode). In addition, in both $\mathrm{D}$ and $\mathrm{SH}$ muscles, the time to peak tension in twitch mode and the twitch to tetanus tension ratio were measured. A muscle contracting isometrically performs no work nor does muscle shortening under zero-load conditions. However, inefficient contractions may be physiologically important when either $P \max$ or higher speed (vZL) are required.

Peak mechanical work and tension/velocity relationship. $W \max$ and the characteristics of the tension/velocity relationship were analysed under tetanic conditions only. Contractions (8-10) were performed, in which the load was regularly incrementally increased from zero up to isometry (fig. 1). For each contraction, the maximum extent of muscle shortening $(\Delta \mathrm{L})$, peak velocity $(\mathrm{v})$ and total isotonic tension level $(P)$ were recorded. $W(P \times \Delta \mathrm{L})$ was calculated over the whole load continuum. Thus $W \max \left(\right.$ in $\mathrm{mJ} \cdot \mathrm{g}^{-1}$ ) and its corresponding tension $(P \mathrm{~W}, \max )$, expressed as a percentage of $P \max$ (fig. 1), were determined. $P \mathrm{~W}$,max reflects optimal loading, i.e. the load level at which the contractile system performs the greatest mechanical work. To ensure that comparisons of $W$ were made at similar relative loads, each experimental $W / P$ relationship was fitted using a five-order polynomial function. The equations of the fitted curves were entered into a computer running TransERA HTBasic Advanced Math Library (TransERA Corporation; Provo, UT, USA), which calculated $W$ at fixed relative tension $(P \mathrm{i}=10,20,30,40,50,60,70,80,90$ and $\left.100 \% P_{\max }\right)$. The $P / \mathrm{v}$ relationship was derived from the $\mathrm{v}$ of afterload contractions plotted against $P$, from zero load up to $P \max$ (fig. 1). Data were fitted using Hill's equation [17]: $(P+\mathrm{a})(\mathrm{v}+\mathrm{b})=(\mathrm{c} P \max +\mathrm{a}) \mathrm{b}$, where $\mathrm{c} P \max$ is the maximum calculated value of $P$ and $-\mathrm{a}$ and $-\mathrm{b}$ are the asymptotes of the hyperbola. The theoretical interest of Hill's equation stems from the fact that the hyperbolic form of the $P / \mathrm{v}$ relationship reflects the thermodynamic properties of the muscle $[17,18]$. The curvature of the $P / \mathrm{v}$ relationship (Hill's hyperbola; $G$ ) is linked to 

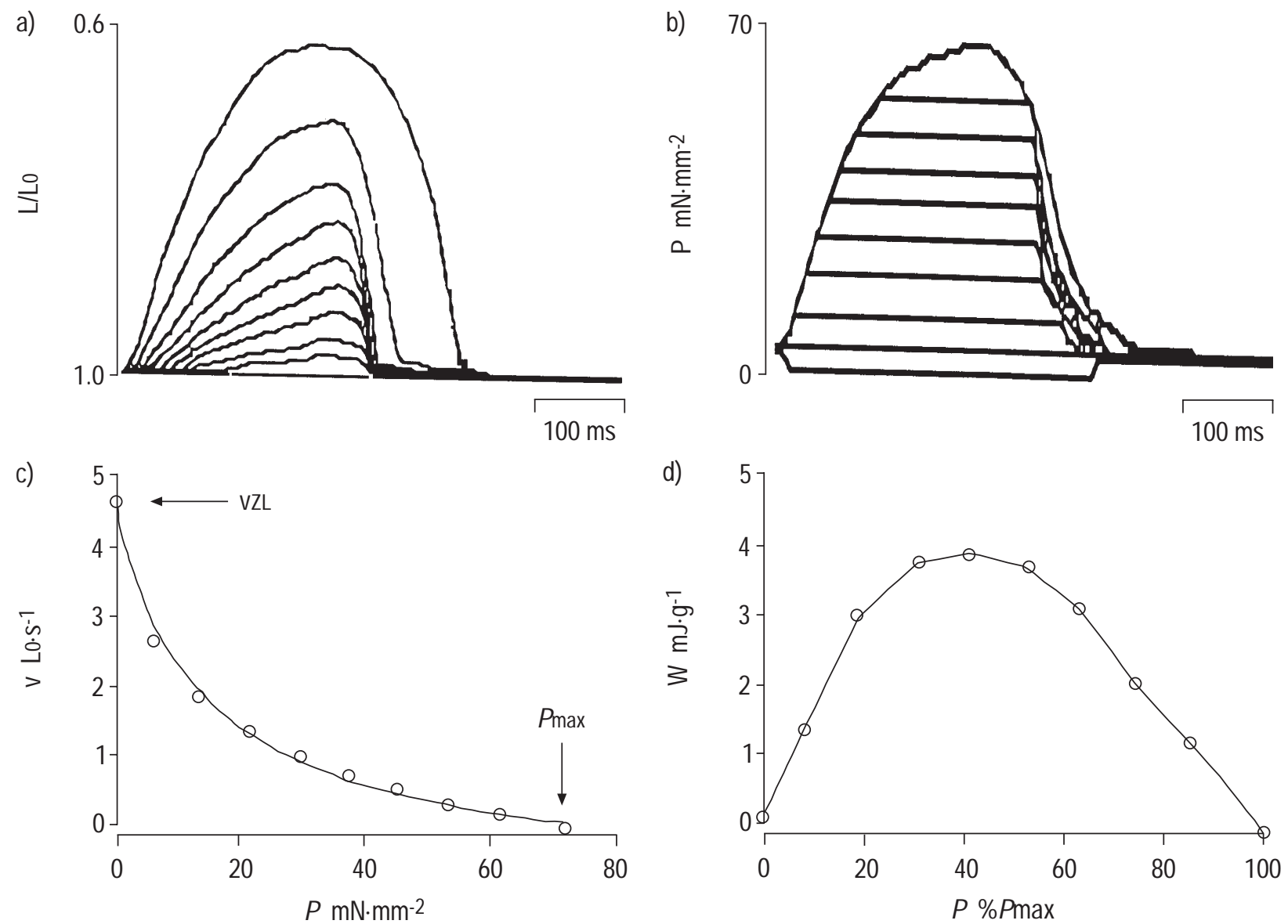

Fig. 1. - Typical recording of sternohyoid muscle under tetanic conditions: a) instantaneous length versus time; b) tension $(P)$ versus time; c) peak velocity (v) versus $P$ and $\mathrm{d})$ mechanical work $(W)$ versus $P$ expressed as a percentage of maximum $(P \max )$. L: muscle length; L0: initial muscle length corresponding to the apex of the length/active $P$ curve; vzL: maximum unloaded shortening velocity.

myothermal economy and cross-bridge kinetics $[18,19]$. It has been shown that $G=\mathrm{c} P \max / \mathrm{a}=\mathrm{cvZL} / \mathrm{b}$, where cvZL is the calculated vZL. Effmax is the maximum ratio of the rate of $W$ to the rate of total energy [19]. It has been shown that effmax $=(G /(G+2))^{2}[20]$.

\section{Fatigue protocol}

Fatigue was induced by repeatedly stimulating each strip with 75 trains $\cdot \mathrm{min}^{-1}$ of $200-\mathrm{ms}$ duration at a stimulation frequency of $50 \mathrm{~Hz}$ under isometric conditions. $P \max$ was monitored and progressively decreased. Stimulation continued until the muscle strip was fatigued to the point where it generated only $\sim 50 \%$ of baseline $P$ max. Then vzL, $G$, effmax, $W$ max and $P$ W,max were determined in tetanic mode using similar methods to those used before fatigue.

\section{Statistical analysis}

Data were expressed as mean \pm SD. Student's unpaired ttest was used to make comparisons between $\mathrm{SH}$ and D muscles. In each muscle group, Student's paired t-test was used to make comparisons between twitch and tetanus, and between baseline and fatigue. Finally, percentage changes in mechanical parameters induced by the fatigue protocol were also calculated. A p-value $<0.05$ was required to reject the null hypothesis.

\section{Results}

\section{Control conditions}

The cross-sectional area of the muscles was $1.14 \pm 0.19$ $\mathrm{mm}^{2}$ in $\mathrm{SH}$ and $1.02 \pm 0.09 \mathrm{~mm}^{2}$ in D muscles.

Under both twitch and tetanic conditions, $P \max$ was lower in SH than in D muscles $(\mathrm{p}<0.05)$. The twitch to tetanus ratio was higher in $\mathrm{D}(0.50 \pm 0.06)$ than in $\mathrm{SH}$ muscles $(0.38 \pm 0.06)(\mathrm{p}<0.001)$. In both SH and D muscles, there was no difference in vzL between twitch and tetanic modes (fig. 2). Under both twitch and tetanic conditions, vZL was higher in SH than in D muscles $(p<0.001)$. In twitch, the time to peak tension was shorter in $\mathrm{SH}(52 \pm 3$ $\mathrm{ms})$ than in D muscles $(70 \pm 6 \mathrm{~ms})(\mathrm{p}<0.001)$.

The $W$ and $P / \mathrm{v}$ relationships were studied in tetanic mode. In both muscles, the $W$ versus $P$ relationship was bell-shaped (figs. 1 and 3 ), indicating a marked influence of loading conditions on mechanical work. The $W \max$ was higher in D than in $\mathrm{SH}$ muscles $(8.0 \pm 2.1$ versus $5.5 \pm 1.2$ $\left.\mathrm{mJ} \cdot \mathrm{g}^{-1} ; \mathrm{p}<0.01\right)$. Moreover, $P \mathrm{~W}, \max$ was higher in $\mathrm{SH}$ $(41 \pm 2.4 \%)$ than in D muscles $(34 \pm 3 \%)(\mathrm{p}<0.001)$. The $G$ and effmax were higher in D than in SH muscles $(G 7.8 \pm$ 1.7 versus $6.1 \pm 1.4, \mathrm{p}<0.05$; effmax: $62.6 \pm 5.8$ versus $56.0 \pm$ $6.9 \% ; \mathrm{p}<0.05$ ), indicating that contraction was more economical in $\mathrm{D}$ than in $\mathrm{SH}$ muscles. 

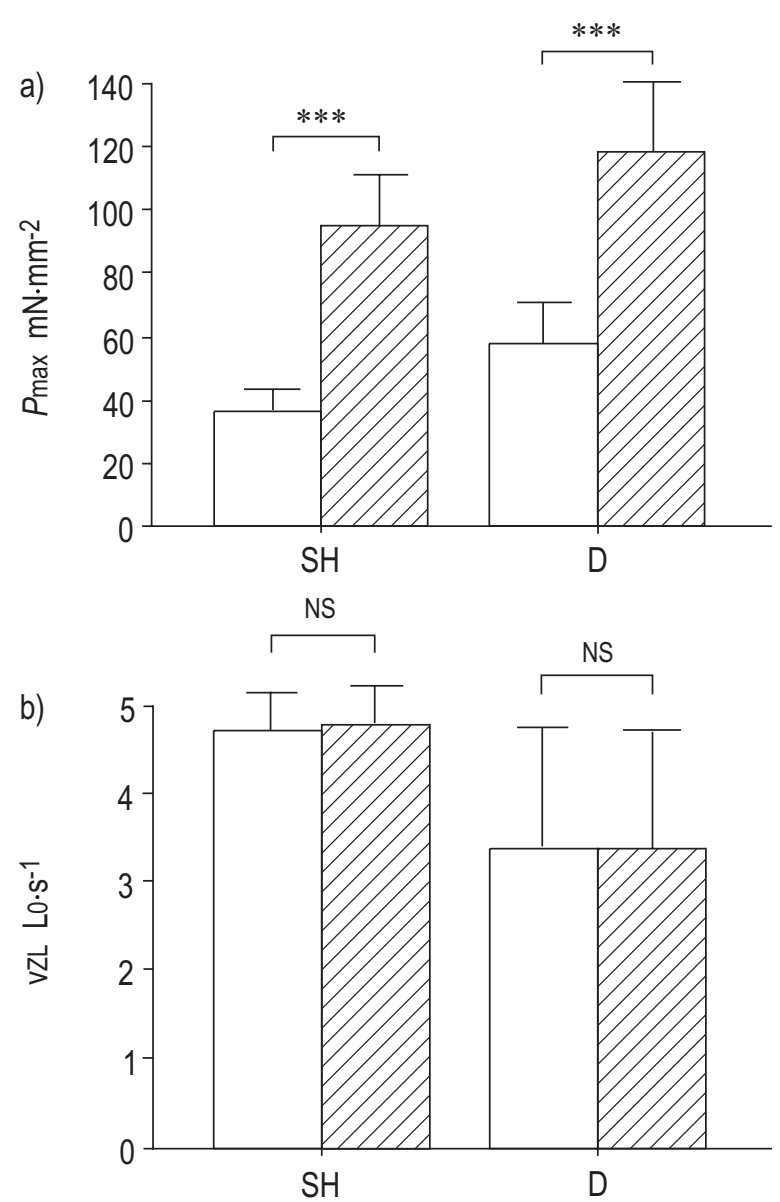

Fig. 2. - a) Maximum isometric tension $(P \max )$; and b) maximum unloaded shortening velocity (vZL) in sternohyoid $(\mathrm{SH})$ and diaphragm (D) muscles under twitch $(\square)$ and tetanic $(\mathbb{Z})$ conditions. Data are presented as mean $\pm \mathrm{SD}(\mathrm{n}=10)$. L0: initial muscle length corresponding to the apex of the length/active $P$ curve; Ns: nonsignificant. ${ }^{* *}: \mathrm{p}<0.001$.

\section{Effects of fatigue}

vZL was significantly reduced in $\mathrm{SH}$ and $\mathrm{D}$ muscles (both $\mathrm{p}<0.001)$ (table 1) when muscles were fatigued to a similar extent ( $\sim 50 \%$ of $P \max )$. In both $\mathrm{SH}$ and D muscles, fatigue also reduced $W_{\max }($ both $\mathrm{p}<0.001)$ (table 1$)$. In $\mathrm{D}$ muscles, $P \mathrm{~W}$,max was unchanged. Conversely, fatigue modified optimal loading in $\mathrm{SH}$ muscles as $P \mathrm{~W}$,max significantly decreased (fig. 3 and table 1$)(\mathrm{p}<0.01)$. In $D$ muscles, fatigue improved the myothermal economy of contraction, as indicated by the significant increase in the $G$ curvature of the $P / \mathrm{v}$ relationship and in the effmax (table 1 ). Conversely, fatigue did not modify $G$ curvature and effmax in SH muscles (table 1).

\section{Discussion}

This is the first report of the isotonic mechanical properties of pharyngeal dilator muscle throughout the overall load continuum. Pharyngeal and D muscles contract and relax in synergy, which is why it was decided to compare their mechanical performance. The results of the present study were as follows: 1) SH muscles had a higher vZL and lower $P_{\max }, W_{\max }$ and effmax than D muscles; 2) $W_{\max }$
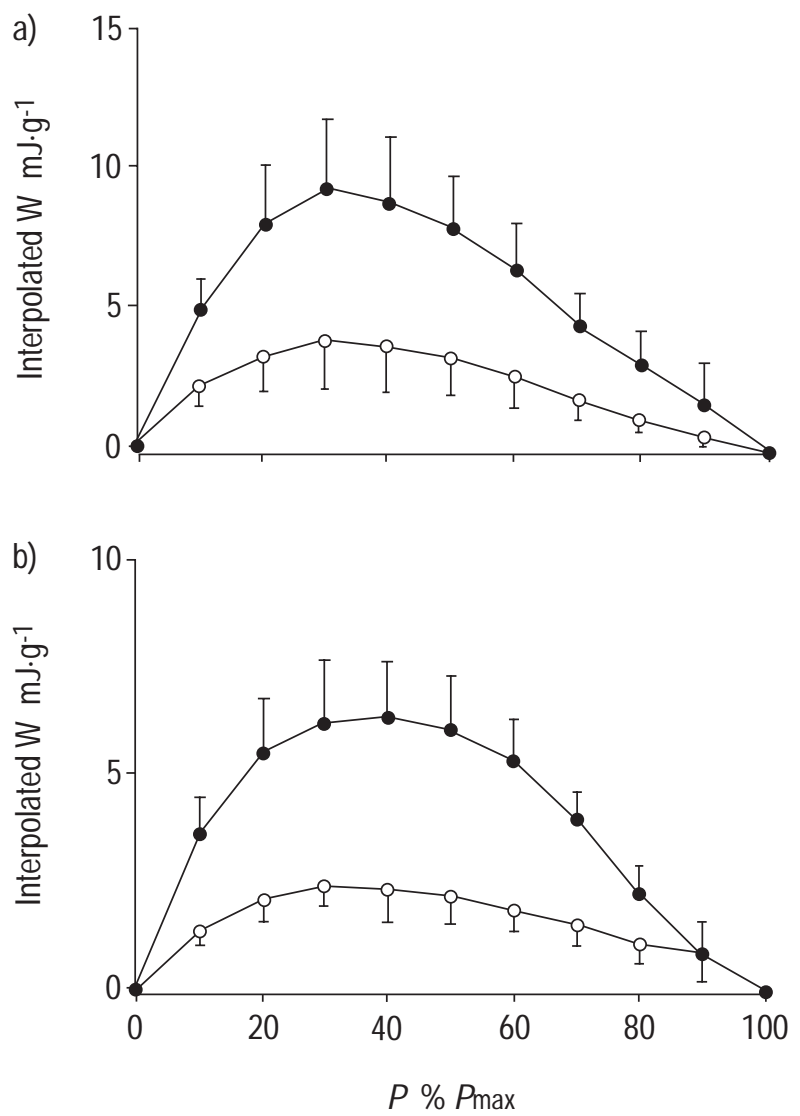

Fig. 3. - Interpolated mechanical work $(W)$ versus tension $(P)$ expressed as a percentage of maximum $\left(P_{\max }\right)$ relationship in: a) diaphragm $(\mathrm{n}=10)$ and $\mathrm{b})$ sternohyoid $(\mathrm{n}=10)$ muscles at baseline $(\boldsymbol{O})$ and during fatigue $(\bigcirc)$. Interpolated $W$ was calculated using a five-order polynomial function (see Methods) to ensure that comparisons of $W$ were made at similar levels of relative load. Data are presented as mean \pm SD.

occurred at a higher relative load in $\mathrm{SH}$ muscles $(40 \%$ $P$ max $)$ than in D muscles $\left(30 \% P_{\max }\right)$; and 3$)$ fatigue did not modify effmax in $\mathrm{SH}$ muscles, whereas it improved effmax in D muscles.

The ability to generate mechanical (i.e. external) work is a key muscle property that determines, at least in part, physiological activity. $\mathrm{SH}$ muscles differed from $\mathrm{D}$ muscles in terms of $W$ max and myothermal economy. $W$ max was higher in the D than in the SH pharyngeal muscle. Importantly both the $G$ curvature of the $P / \mathrm{v}$ relationship and the effmax were higher in D than in SH muscles, attesting to more

Table 1. - Effects of fatigue on sternohyoid (SH) and diaphragm (D) muscles

\begin{tabular}{lcc}
\hline & $\mathrm{SH}$ & $\mathrm{D}$ \\
\hline Muscles n & 10 & 10 \\
vZL & $-16 \pm 6^{* * *}$ & $-24 \pm 7^{* * *}$ \\
$W \max$ & $-62 \pm 5^{* * *}$ & $-60 \pm 4^{* * *}$ \\
$P \mathrm{~W}, \max$ & $-13 \pm 9^{* *}$ & $-2 \pm 16$ \\
$G$ & $-8 \pm 22$ & $29 \pm 26^{*}$ \\
effmax & $-6 \pm 13$ & $10 \pm 9^{*}$ \\
\hline
\end{tabular}

Data are presented as mean \pm SD percentage change. vZL: maximum unloaded shortening velocity; $W$ max: peak mechanical work; $P$ W,max: tension at which $W \max$ occurred; $G$ : curvature of Hill's hyperbola; effmax: peak mechanical efficiency. ${ }^{*}: \mathrm{p}<0.05$, $* *: \mathrm{p}<0.01, * * *: \mathrm{p}<0.001$ versus baseline. 
economical contraction in D than in SH muscles [18]. In other words, as compared to $\mathrm{SH}$ muscles, greater $W$ was achieved in D muscles at a lower energetic cost. In both muscles, $W$ was strongly dependent on loading conditions. $W \max$ occurred at a lower relative load $(P \mathrm{~W}, \max )$ in D than in SH muscles (fig. 3). This suggests that subtle changes in loading conditions may optimize work differently in $\mathrm{SH}(P \mathrm{~W}, \max \sim 40 \% P \max )$ and in $\mathrm{D}$ muscles $\left(P \mathrm{~W}, \max \sim 30 \% P_{\max }\right)$.

vZL was higher in SH than in D muscles. This is in agreement with the fact that rat $\mathrm{D}$ and $\mathrm{SH}$ muscles have different fibre-type composition. Rat SH muscle is composed essentially of fast (type II) fibres (>90\%) [21-23] whereas rat $\mathrm{D}$ is a mixed fibre muscle, containing $40 \%$ slow fibres (type I) $[22,23]$. Moreover, time to peak tension was shorter in $\mathrm{SH}$ than in D muscles, in accordance with results in the literature [10]. Pmax was higher in D than in SH muscles. Even though there is some controversy regarding the existence of differences in $P$ max between type I and type II fibres, a higher $P \max$ in SH than in D muscles would still have been expected [24]. Yet, the contrary was observed. This could be explained by differences in fibre orientation or in the number and/or unit force of cross-bridges in the two different muscles. Indeed, it is widely accepted that $P$ max mainly reflects the number of working actomyosin cross-bridges [13]. Thus, the present results indirectly suggest differences in the number of cross-bridges between isometrically contracting SH and D muscles. Although further studies are needed to confirm this, the higher efficiency in D than in SH muscles is also consistent with a higher single force of cross-bridges in D muscles $[14,15]$.

The present study also compared mechanics and energetics after fatigue. The fatigue experiments were carried out when the decrease in $P \max$ was similar in the two muscles $(\sim 50 \%)$. Fatigue induced similar falls in vZL $(\sim 20 \%)$ and $W_{\max }(\sim 60 \%)$. Conversely, changes in myothermal economy differed markedly between the two muscles. Fatigue improved the economy of contraction in D muscles, as reflected by the increase in effmax and $G$ (table 1$)$. In contrast, fatigue did not modify effmax and $G$ in SH muscles. Previous studies have shown that fatigue improves the economy of force generation in mixed-fibre muscle (e.g. rat D), whereas it does not change economy in muscle mainly composed of one fibre type $[25,26]$. Differences in myosin fibre composition between rat D and SH pharyngeal muscle are likely to explain the diferent effects of fatigue on muscle energetics observed in this study. It is well known that fast-twitch fibres fatigue more quickly than slow-twitch fibres [27, 28]. Thus, increased effmax in rat $\mathrm{D}$ could be explained by a predominant effect of fatigue on fast (fatigue-sensitive) fibres, thus shifting the overall characteristics of the muscle towards those of slow (fatigue-resistant) fibres [25]. Conversely, in fastfibre muscles such as pharyngeal muscles, no preferential fibre-type recruitment occurs, which might partly explain why effmax and $G$ did not change significantly during fatigue (table 1). Recently, it has been suggested that pharyngeal dilator muscles may fail in the presence of increased load [23].

In fatigued $\mathrm{D}, P \mathrm{~W}$,max did not change as effmax increased (table 1). Thus, the fatigued rat D worked under more economical conditions. In contrast, the present study suggests less favourable mechanical behaviour of $\mathrm{SH}$ as compared to D muscles. Indeed, in SH muscles: 1) effmax did not increase during fatigue (table 1); and 2) $P$ w,max was shifted towards a lower load level. Thus, for a given degree of fatigue, the $\mathrm{SH}$ pharyngeal muscle operated under less favourable energetic conditions than the D, especially when the load placed on the muscle was increased. This might help explain why pharyngeal dilator muscles may fail in the presence of increased load.

It is important to emphasize that the purpose of this study was not to test the fatiguability of the two different muscles. The fatiguability of pharyngeal muscles has been studied in rat and cat $[10,11]$. In these studies, the muscles have been fatigued by using the protocol of BURKE et al. [29], in which the decrease in $P \max$ is plotted as a function of time. Fatiguability differs among species; as compared to the $\mathrm{D}$, the pharyngeal musculature is relatively fatigueresistant in cats and fatigue-sensitive in rats.

\section{Limitations}

The results pertain strictly to the animal species and experimental conditions used. Species differences in mechanical properties of pharyngeal muscles have been carefully investigated under isometric conditions [9-11], and the possibility that other species have different mechanical and energetic isotonic properties cannot be excluded. This study was carried out at $22^{\circ} \mathrm{C}$ so as to improve the stability of isotonic indices. The results can thus only be extrapolated to in vivo conditions with great care, given that temperature influences muscle performance [12].

\section{Clinical implications}

The co-ordinated activity of $\mathrm{D}$ and pharyngeal muscles helps maintain normal pharyngeal patency during breathing. The different responses to fatigue observed in D and SH muscles may modify the mechanical equilibrium between $\mathrm{D}$ and pharyngeal muscles and may promote respiratory disturbances. In sleep apnoea syndrome, occlusions of the upper airways occur many times during sleep. A relative decline in the activity of pharyngeal dilator muscles seems to be associated with the occlusions [7]. The end of occlusions is associated with large bursts of genioglossus activity. The acute effects of these repeated episodes of pharyngeal dilator muscle recruitment are unknown. The results obtained after fatigue suggest that a vicious circle might be produced during fatigue-like periods, the hyperactivity of pharyngeal muscles placing them under disadvantageous mechanical and energetic conditions. This may precipitate pharyngeal muscle failure and then promote occlusion of the upper airways. Further studies are needed to confirm this hypothesis. Lastly, electrical stimulation of pharyngeal muscles during sleep has been proposed in the treatment of sleep apnoea syndrome [30]. The present approach may help to evaluate the effects of electrical stimulation on the isotonic properties of pharyngeal muscles.

\section{Conclusion}

To summarize, this study reports on the isotonic mechanical properties of rat sternohyoid and diaphragm muscles 
at baseline and during fatigue. Compared to the diaphragm, the mechanical performance of the sternohyoid muscle was lower and the economy of contraction was less efficient. During fatigue, the sternohyoid pharyngeal muscle exhibited unfavourable mechanical behaviour, from a thermoenergetic point of view, as compared to the diaphragm.

\section{References}

1. Van Lunteren E, Strohl KP. The muscles of the upper airways. Clin Chest Med 1986; 7: 171-188.

2. Frugière A, Barillot JC. Respiratory-related activity of pharyngeal nerves in the rat. Respir Physiol 1994; 98: 295-304.

3. Sauerland EK, Harper RM. The human tongue during sleep: electromyographic activity of the genioglossus muscle. Exp Neurol 1976; 51: 160-170.

4. Wiegand DA, Latz B, Zwillich CW, Wiegand L. Geniohyoid muscle activity in normal men during wakefulness and sleep. J Appl Physiol 1990; 69: 1262-1269.

5. Sherrey JH, Pollard MT, Megirian D. Respiratory functions of the inferior pharyngeal constrictor and sternohyoid muscles during sleep. Exper Neurol 1986; 92: 267-277.

6. Kuna ST, Vanoye CR. Respiratory-related pharyngeal constrictor muscle activity in decerebrate cats. $J$ Appl Physiol 1997; 83: 1588-1594.

7. Remmers JE, Degroot WJ, Sauerland EK, Anch AM. Pathogenesis of upper airway occlusion during sleep. $J$ Appl Physiol 1978; 44: 931-938.

8. Mathew OP. Upper airway negative-pressure effects on respiratory activity of upper airway muscles. $J \mathrm{Appl}$ Physiol 1984; 56: 500-505.

9. Salomone RJ, Van Lunteren E. Effects of aging on the physiological characteristics of pharyngeal dilator muscle and diaphragm in rats. Am Rev Respir Dis 1991; 143: A563.

10. Van Lunteren E, Vafaie H. Force potentiation in respiratory muscles: comparison of diaphragm and sternohyoid. Am J Physiol 1993; 264: R1095-R1100.

11. Van Lunteren E, Vafaie H, Salomone RJ. Comparative effects of aging on pharyngeal and diaphragm muscles. Respir Physiol 1995; 99: 113-125.

12. Metzger JM, Scheidt JB, Fitts RH. Histochemical and physiological characteristics of the rat diaphragm. $J$ Appl Physiol 1985; 58: 1085-1091.

13. Huxley AF. Muscle structure and theories of contraction. Prog Biophys Biophys Chem 1957; 7: 255-318.

14. Coirault C, Lambert F, Joseph T, Blanc FX, Chemla D, Lecarpentier Y. Developmental changes in crossbridge properties and myosin isoforms in hamster diaphragm. Am J Respir Crit Care Med 1997; 156: 959-967.

15. Lecarpentier Y, Chemla D, Blanc FX, et al. Mechanics, energetics, and crossbridge kinetics of rabbit diaphragm during congestive heart failure. FASEB J 1998; 12: 981989.
16. Brutsaert DL, Claes VA, Goethal MA. Effect of calcium on force-velocity-length relations of heart muscle of the cat. Circ Res 1973; 32: 385-392.

17. Hill AV. The heat of shortening and the dynamic constants of muscle. Proc R Soc Lond Biol Sci 1938; 126: 36-195.

18. Lecarpentier Y, Bugaisky LB, Chemla D, et al. Coordinated changes in contractility, energetics, and isomyosins after aortic stenosis. Am J Physiol 1987; 252: H275$\mathrm{H} 282$.

19. Woledge RC, Curtin NA, Homsher E. Energetic aspects of muscle contraction. Monogr Physiol Soc 1985; 41: 27117.

20. Coirault C, Riou B, Bard M, Suard I, Lecarpentier Y. Contraction, relaxation, and economy of force generation in isolated human diaphragm muscle. Am J Respir Crit Care Med 1995; 152: 1275-1283.

21. Petrof BJ, Kelly AM, Rubinstein NR, Pack AI. Effect of hypothyroidism on myosin heavy chain expression in rat pharyngeal dilator muscles. J Appl Physiol 1992; 73: 179-187.

22. Salomone RJ, Dick TE, Van Lunteren E. Fiber subtype and capillary distributions of pharyngeal dilator muscle and diaphragm in young and old rats. Am Rev Respir Dis 1990; 141: A366.

23. Bracher A, Coleman R, Schnall R, Oliven A. Histochemical properties of upper airway muscles: comparison of dilator and nondilator muscles. Eur Respir J 1997; 10: 990-993.

24. Bottinelli R, Schiaffino S, Reggiani C. Force-velocity relations and myosin heavy chain isoform compositions of skinned fibres from rat skeletal muscle. J Physiol 1991; 437: 655-672.

25. Coirault C, Chemla D, Péry-Man N, Suard I, Lecarpentier Y. Effects of fatigue on force-velocity relation of diaphragm: energetic implications. Am J Respir Crit Care Med 1995; 151: 123-128.

26. Hatcher DD, Luff AR. Force-velocity properties of fatigue-resistant units in cat fast-twitch muscle after fatigue. J Appl Physiol 1987; 63: 1511-1518.

27. Burke RE, Levine DN, Zajac FE III, Tsaris P, Engel WK. Mammalian motor units: physiological-histochemical correlation in three types in cat gastrocnemius. Science 1971; 174: 709-712.

28. Lieberman DA, Faulkner JA, Craig AB, Maxwell LC. Performance and histochemical composition of guinea pig and human diaphragm. J Appl Physiol 1973; 34: 233237.

29. Burke RE, Levine DN, Tsaris P, Zajac FE III. Physiological types and histochemical profiles in motor units of the cat gastrocnemius. J Physiol Lond 1973; 234: 723748.

30. Miki H, Hida W, Chonan T, Kikuchi Y, Takishima T. Effects of submental electrical stimulation during sleep on upper airway patency in patients with obstructive sleep apnoea. Am Rev Respir Dis 1989; 140: 1285-1289. 